In der Rubrik „Literatur kompakt" werden die wichtigsten Originalarbeiten aus der internationalen Fachliteratur referiert.

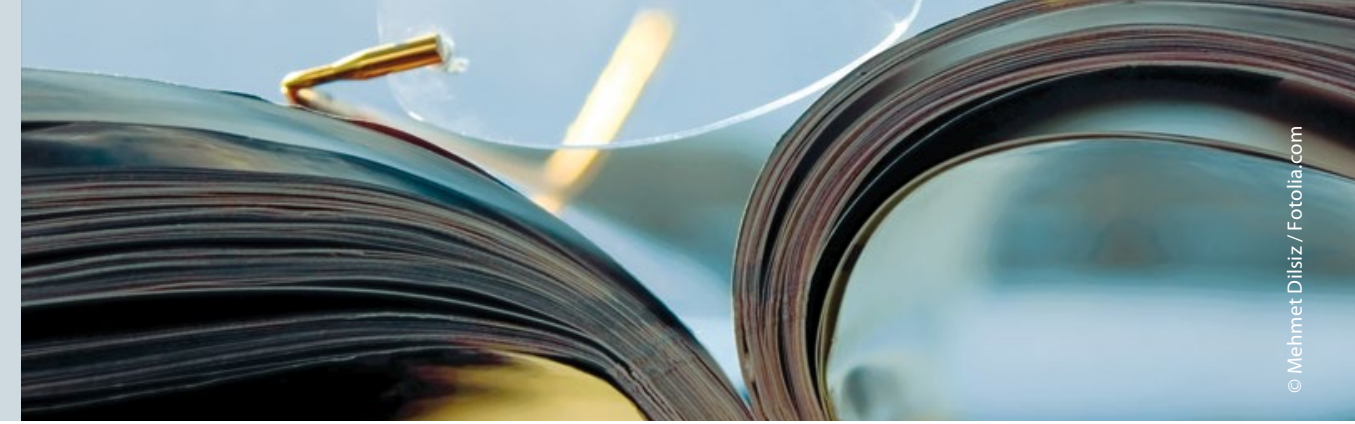

\section{Erhöhtes OP-Risiko bei Migräne}

\begin{abstract}
Migränepatienten, vor allem solche mit Aura, haben kurz nach einer OP ein deutlich erhöhtes Schlaganfallrisiko. Das hat eine prospektive Registerstudie ergeben. Ein internationales Ärzteteam fordert daher, die Migräne in die perioperative Risikobewertung aufzunehmen.
\end{abstract}

$\mathrm{D}$ ass zwischen Migräne und dem Auftreten eines Schlaganfalls eine deutliche Assoziation besteht, haben bereits mehrere Beobachtungsstudien nahegelegt. Aktuell hat ein Forscherteam aus den USA, Deutschland und Dänemark Belege dafür gefunden, dass Migränepatienten nach einer Operation in besonderem Maße gefährdet sind, einen ischämischen Insult zu erleiden, vor allem dann, wenn die Kopfschmerzen von einer Aura begleitet sind. Auch die Rate neuerlicher Klinikeinweisungen war bei diesen Patienten deutlich erhöht.

Wie Fanny P. Timm vom Massachusetts General Hospital und Kollegen anhand des lokalen Klinikregisters gezeigt haben, lag die Gesamtinzidenz der Schlaganfälle innerhalb von 30 Tagen nach einem chirurgischen Eingriff bei $0,6 \%$. Nach Abgleichung verschiedener Risikofaktoren schätzten die Forscher die absolute Inzidenz auf insgesamt 2,4 ischämische Schlaganfälle pro 1.000 Chirurgiepatienten. Das Risiko kletterte auf 4,3 pro 1.000 bei Migränepatienten und auf 6,3 pro 1.000 , wenn man nur Migränepatienten mit Aura herausgriff.

In die prospektive Registerstudie waren Daten von 124.558 chirurgischen $\mathrm{Pa}$ tienten eingeflossen, darunter $10.179 \mathrm{mit}$ ärztlich diagnostizierter Migräne. Von den 771 Patienten, die in Folge der OP einen Schlaganfall erlitten hatten, wiesen 89 (11,5\%) eine Migräne in der Ana- mnese auf, davon 18 mit und 71 ohne Aura. Die Migränediagnose erhöhte die Wahrscheinlichkeit für einen perioperativen ischämischen Schlaganfall innerhalb eines Monats signifikant (Odds Ratio [OR]: 1,75). Dabei war das Insultrisiko für Migränepatienten mit Aura deutlich höher als ohne Aura (OR: 2,61 bzw. 1,62).

In $46,2 \%$ der Fälle ereignete sich der Insult in den ersten beiden Tagen nach der Operation. Ein gutes Viertel trat dagegen erst nach der Entlassung auf; auch hierfür war die Wahrscheinlichkeit bei Migränepatienten besonders stark erhöht (OR: 1,94).

Was auffiel, war der Zusammenhang mit einer intraoperativen Verabreichung von Vasopressoren; auch diese scheinen für sich genommen das Schlaganfallrisiko bei Migränepatienten zu erhöhen.

Die Migräne wirkte sich zudem erheblich auf die Rate der erneuten Klinikeinweisungen aus: Insgesamt mussten 10.088 Studienteilnehmer innerhalb von 30 Tagen nach der Entlassung ein zweites Mal in die Klinik. Unter den Migränepatienten war dieses Risiko um $31 \%$ erhöht. Besonders hoch war der Anteil der Zweiteinweisungen bei den Patienten mit Aura (OR: 1,59).

Für den nachbetreuenden Arzt interessant: Bei den erneuten Einweisungen spielten neben neurologischen und kardiovaskulären Erkrankungen auch Er- krankungen des Verdauungstrakts und Symptome wie Übelkeit oder Erbrechen gerade bei Migränepatienten eine maßgebliche Rolle.

Darüber, was das erhöhte Insultrisiko speziell bei Migränepatienten ausmacht, können Timm et al. nur spekulieren: Erstere reagierten möglicherweise besonders empfindlich auf zerebrale Durchblutungsstörungen. Den Forschern zufolge führt hier die Ischämie $\mathrm{zu}$ fortschreitenden Depolarisierungen, was zu einer verlängerten Vasokonstriktion und verringerter Sauerstoffversorgung führen könne. Hinzu kämen möglicherweise Mikroembolien auf der Basis von Shuntanomalien in Lunge oder Herz. Mit der OP in Zusammenhang stehende Prozesse wie die Allgemeinanästhesie, Dehydrierung sowie das perioperative Absetzen von Antikoagulanzien täten ein Übriges, um die Ischämie und damit die Neigung zur Gerinnselbildung zu erhöhen.

Fazit: Präventiv ist es den Autoren zufolge ratsam, gerade bei Migränepatienten Vasopressoren möglichst zurückhaltend einzusetzen und vielmehr während der Anästhesie penibel auf einen adäquaten Volumenersatz zu achten. Die Forscher fordern vor allem, bei der perioperativen Risikobewertung routinemäßig abzuklären, ob der Patient an einer Migräne leidet. Angesichts des deutlich erhöhten Schlaganfallrisikos dieser Patienten sei dies von hoher klinischer Relevanz.

Dr. Elke Oberhofer

Timm FP et al. Migraine and risk of perioperative ischemic stroke and hospital readmission: hospital based registry study. BMJ 2017 Jan 10;356:i6635 